\title{
Analysis of the Risk Factors of Soft Tissue Bleeding Phenomenon in Covid-19 Patients: the Point of View After Two Years of the Pandemic.
}

Eliodoro Faiella ( $\nabla$ e.faiella@policlinicocampus.it)

Campus Bio-Medico University Hospital: Policlinico Universitario Campus Bio-Medico https://orcid.org/0000-0002-7367-6891

\section{Gennaro Castiello}

Campus Bio-Medico University: Universita Campus Bio-Medico di Roma

\section{Domiziana Santucci}

Campus Bio-Medico University: Universita Campus Bio-Medico di Roma

\section{Carlo Altomare}

Campus Bio-Medico University: Universita Campus Bio-Medico di Roma

\section{Giuseppina Pacella}

Campus Bio-Medico University: Universita Campus Bio-Medico di Roma

\section{Caterina Bernetti}

Campus Bio-Medico University: Universita Campus Bio-Medico di Roma

\section{Ugo Ferrari}

Campus Bio-Medico University: Universita Campus Bio-Medico di Roma

Moises Muley Villamu

Campus Bio-Medico University: Universita Campus Bio-Medico di Roma

Raffaele Antonelli Incalzi

Campus Bio-Medico University: Universita Campus Bio-Medico di Roma

Bruno Beomonte Zobel

Campus Bio-Medico University: Universita Campus Bio-Medico di Roma

\section{Rosario Francesco Grasso}

Campus Bio-Medico University: Universita Campus Bio-Medico di Roma

\section{Research Article}

Keywords: Coronavirus disease (COVID-19), soft tissue bleeding phenomenon, patients, pandemic

Posted Date: January 20th, 2022

DOI: https://doi.org/10.21203/rs.3.rs-1247099/v1 
License: (c) (i) This work is licensed under a Creative Commons Attribution 4.0 International License. Read Full License 


\section{Abstract}

Background: to analyze the relationship between patient characteristics, including anagraphic, laboratoristic data and amount of adipose tissue measured in Computed Tomography (CT)-scans in COVID-19 patients, and incidence of soft-tissue bleeding requiring medical and/or interventional radiology management. A total of 132 patients hospitalized for COVID-19 pathology from October 2020 to May 2021, were included in the study, considering two groups: a bleeding group, 70 cases of softtissue bleeding occurred during hospitalization, and a control group, 62 hospitalized COVID-19 patients without bleeding events. In the bleeding group, two subgroups were considered: an embolization group including soft tissue bleeding cases requiring interventional radiology TAE (Transarterial Embolization) (16/70; 22.9\%) and a non-embolization group, clinically managed without TAE (54/70; 77.1\%).

Demographics and clinical data, visceral adipose tissue (VAT), sub-cutaneous adipose tissue (SAT) areas measured on CT images and VAT/SAT ratio were compared between bleeding and control groups and between embolization and non-embolization subgroups.

Results: Bleeding and control groups did not significantly differ for sex distribution, Covid-19, platelet count, SAT-area, VAT-area and VAT/SAT ratio. Embolization and non-embolization groups did not significantly differ for age, Covid-19, platelet count, SAT area, and VAT/SAT ratio. A statistically significant difference was observed between embolization and non-embolization groups for VAT area, with smaller values in embolization group (mean difference: $64.2 \mathrm{~cm}^{2}, 95 \% \mathrm{Cl}, 8.3-120.1 ; \mathrm{p}<0.05$ ).

Conclusions: Soft-tissue bleeding in COVID-19 is more frequent and severe in patients with low amount of visceral adipose tissue, demonstrating that fat mass may have a containing function on bleeding, limiting its progression in surrounding structures. Other factors influence the risk of bleeding, such as age.

\section{Introduction}

Coronavirus disease (COVID-19) caused by Severe Acute Respiratory Syndrome Coronavirus 2 (SARSCoV-2) was declared a pandemic by The World Health Organization (WHO) for its huge impact on health systems[1]. Respiratory system involvement is considered the principal cause of death in patients with COVID-19[2], [3] although changes in coagulation function have been also commonly associated with SARS-CoV-2 infection as one of the most important adverse prognostic signs[4], [5]. Dysregulation of coagulation caused by host response to viral replication produces an hypercoagulability state often resulting in venous and arterial thrombosis and multiorgan dysfunction[6]. Consequently, heparin-based therapies were introduced[7] which, together with factors such as thrombocytopenia, hyperfibrinolytic state and consumption of coagulation factors, produce a higher risk of bleedings[8]-[10] in patients affected by COVID-19, including soft tissue hematomas requiring medical or interventional radiology management through trans-arterial embolization (TAE)[11]. Several studies showed a strong association between obesity and COVID-19 severity[12]-[17]. Obese patients affected by COVID-19 demonstrated an overall worse disease outcome with more severe infection forms, higher hospitalization and mechanical 
ventilation rates[18] compared to non-obese subjects. COVID-19 coagulopathy is also aggravated by obesity due to its intrinsic coagulation alterations that are in addition to those with those related to the SARS-CoV-2 infection[19]. In particular, excess visceral fat is considered an important risk factor for COVID-19 severity due to its viral receptor and cytokine expression[20]. The aim of this study is to analyze the relationship between patient characteristics, including anagraphic data, laboratoristic data and the amount of adipose tissue measured in CT scans in COVID-19 patients, and the incidence of soft tissue bleeding requiring medical and/or interventional radiology management.

\section{Materials And Methods Study population}

The study was conducted according to the guidelines of the Declaration of Helsinki. Ethical review and approval were waived for this study, due to retrospective nature of the study. Informed Consent Statement was obtained from all subjects involved in the study at the time of CT execution.

A total of 132 patients hospitalized at out center for COVID-19 pathology from October 2020 to May 2021, were included in the study, considering two groups: a bleeding group, including 70 cases of soft tissue bleeding occurred during hospitalization, and a control group, with 62 hospitalized COVID-19 patients without bleeding events. In the bleeding group, two subgroups were considered: an embolization group including soft tissue bleeding cases which required interventional radiology treatment with TAE $(16 / 70 ; 22.9 \%)$ and a non-embolization group of soft tissue bleeding cases which were clinically managed without TAE (54/70; 77.1\%).

\section{Soft tissue bleeding management}

In cases of soft tissue bleeding, conservative management was the main strategy for hemodynamically stable patients. TAE was performed in cases of progressive, uncontrolled bleeding (confirmed by CT angiography) leading to hemodynamic instability. Embolizing techniques included coils alone, glue alone, gelfoam alone and combinations of microparticles with coils, gelfoam and coils, glue and coils (Figures 1 and 2). Bleeding sites and embolization techniques are listed in Table 1. 
Table 1

Soft tissue bleeding features and embolization techniques.

\begin{tabular}{|ll|}
\hline STB features & $n$ \\
\hline Bleeding site & 16 \\
\hline Iliopsoas muscle & 12 \\
\hline Rectus abdominis muscle & 10 \\
\hline Lower limb muscles & 8 \\
\hline Upper limb muscles & 8 \\
\hline Upper abdomen & 6 \\
\hline Pelvic floor & 6 \\
\hline Thorax muscles & 2 \\
\hline Masseter muscle & 2 \\
\hline Neck muscles & \\
\hline Embolization technique & 4 \\
\hline Coils & 2 \\
\hline Glue & 2 \\
\hline Gelfoam & 2 \\
\hline Coils + gelfoam & 2 \\
\hline Coils + glue & 4 \\
\hline Coils + microparticles & \\
\hline
\end{tabular}

\section{Data collection}

Demographics and clinical data were collected including platelet count (PLT) and hemoglobin levels (Hb) at the patient admission and pulmonary involvement severity score (SS)[21], [22]. We also counted how many days after admission bleeding occurred, estimating the time of admission to the hospital with the start of anticoagulant therapy. At least one CT examination was performed in all patients during hospitalization, with contrast medium administration, using a Dual Source 384-slice (2x192) CT (Siemens SOMATOM Force, Erlangen, Germany) with real-time voltage modulation (70-150 kV), real-time dose modulation (CARE Dose4D ${ }^{\mathrm{Tm}} ; 80-250 \mathrm{mAs}$ ), spiral pitch factor of 1.8 and collimation width of $0.6 \mathrm{~mm}$. Measurements of subcutaneous adipose tissue (SAT) and visceral adipose tissue (VAT) areas in $\mathrm{cm}^{2}$ 
were obtained analyzing axial CT images at the level of the third lumbar vertebra with an image processing application (OsiriX, Pixmeo, Bernex, Switzerland)(Figure 3)[23].

\section{Statistical analysis}

Demographics and clinical data, VAT, SAT areas and the VAT/SAT ratio were compared between the bleeding and the control groups using Chi-Square and Student's $t$ test. The same variables were then compared between the embolization and non-embolization subgroups using Chi-Square and Student's $\mathrm{t}$ test. Statistical significance was considered as $p<0.05$. Statistical analysis was performed using IBM SPSS Statistics for Windows, version 26 (IBM Corp., Armonk, N.Y., USA).

\section{Results}

The bleeding group consisted of 70 cases while the control group included 62 cases. Bleeding never occurred before 4 days after admission, with a mean of onset of 8 days. Bleeding and control groups did not significantly differ for sex distribution, Covid-19 SS, PLT count, SAT area, VAT area and VAT/SAT ratio although SAT and VAT areas tend to be lower in the bleeding group, although in a non-significant way. There was a slight significant difference in age between bleeding group and control group, with older patients in the former and a mean difference of 6 years $(95 \% \mathrm{Cl}, 0.4-11.6 ; \mathrm{p}<0.05)$.

The embolization group consisted of 16 cases while the non-embolization group included 54 cases. Embolization and non-embolization groups did not significantly differ for age, Covid-19 SS, PLT count, SAT area, and VAT/SAT ratio. There was a significant difference in sex between embolization and nonembolization groups, with a higher number of females compared to male in the former group $(p=<0.05)$. A statistically significant difference was observed between the embolization and non-embolization groups for VAT area, with smaller values in the embolization group and a mean difference of $64.2 \mathrm{~cm}^{2}(95 \% \mathrm{Cl}$, $8.3-120.1 ; p<0.05)$. Results are listed in Tables 2 and 3.

Table 2

Variables values for bleeding group and control group.

\begin{tabular}{|llll|}
\hline Variables & Bleeding group & Control group & $p$ \\
\hline$N$ & 70 & 62 & \\
\hline Sex (male; female) & $50 ; 20$ & $46 ; 16$ & $>0.05$ \\
\hline Age (mean) \pm SD & $70.9 \pm 11,6$ & $65 \pm 11.2$ & $<0.05$ \\
\hline Covid-19 SS & $12.5 \pm 4.7$ & $14.6 \pm 4.5$ & $>0.05$ \\
\hline PLT & $235.3 \pm 91.2$ & $254 \pm 75.6$ & $>0.05$ \\
\hline SAT & $136 \pm 78$ & $159 \pm 82.2$ & $>0.05$ \\
\hline VAT & $168 \pm 84.6$ & $196 \pm 101.9$ & $>0.05$ \\
\hline VAT/SAT & $1.5 \pm 0.8$ & $1.5 \pm 0.9$ & $>0.05$ \\
\hline
\end{tabular}


Table 3

Variables values for embolization group and non-embolization group.

\begin{tabular}{|llll|}
\hline Variables & Embolization group & Non-Embolization group & $\boldsymbol{p}$ \\
\hline$n$ & 16 & 54 & \\
\hline Sex (male; female) & $6 ; 10$ & $44 ; 10$ & $<0.05$ \\
\hline Age (mean) \pm SD & $72.6 \pm 6.2$ & $70.4 \pm 12.8$ & $>0.05$ \\
\hline Covid-19 SS & $10.1 \pm 4.6$ & $13.2 \pm 4.5$ & $>0.05$ \\
\hline PLT & $276.1 \pm 65.7$ & $222.7 \pm 95.2$ & $>0.05$ \\
\hline SAT & $105.6 \pm 63.2$ & $145 \pm 80.7$ & $>0.05$ \\
\hline VAT & $118.5 \pm 58.4$ & $182.7 \pm 86.4$ & $<0.05$ \\
\hline VAT/SAT & $1.4 \pm 0.7$ & $1.6 \pm 0.8$ & $>0.05$ \\
\hline
\end{tabular}

\section{Discussion}

The bleeding phenomenon in patients affected by Covid-19 is of crucial importance for their clinical management and prognosis[6], [9], [10]. The bleeding events are the results of a series of clinical and therapeutic factors depending on the intrinsic characteristics of the infection and the standard therapeutic strategies implemented to manage the occurrence of thrombotic events[8]. Given the importance of the association between obesity and the clinical outcome in patients with Sars-Cov-2 infection[13], [14], [18], we aimed to analyze the relationship between the onset of soft tissue bleeding events and the amount of adipose tissue measured through CT scan analysis. We considered the need for TAE treatment as an additional factor to determine the complexity of soft tissue bleeding[11]. Our results show that, although factors such as age are related to a higher risk of soft tissue bleeding events according to an overall increased risk of worse prognosis in elder people[3], bleeding in Covid-19 patients is not dependent on the amount of adipose tissue. In our population, a lower amount of VAT in patients with soft tissue bleeding who required TAE was observed, compared to patients undergoing medical treatment alone. Even more, a tendency to a lower amount of VAT and SAT in patients with bleeding compared to the control group was counted. Literature data show a worse overall prognosis of obese patients or those with greater amounts of visceral fat, presumably determined by inflammatory and metabolic factors[20], [24]. Our work shows that soft tissue bleeding phenomenon occurs most frequently in elderly patients[25] and that unstable bleedings requiring TAE are most frequent in patients with lower VAT. We hypothesize that complicated bleedings have a pathogenesis mechanism related to microtraumatic injuries to soft tissues rather than the patient's fat mass. It is well known that non-self limiting bleedings are more frequently associated with muscle lesions[26], [27], often caused by the only mobilization on Covid-19 patients, on which anticoagulant therapy is an additive risk factor[28], [29].We assume that, most likely, a greater fat mass can have a containing function on bleeding by limiting its 
progression in surrounding structures. In conclusion, our data suggest that soft tissue haemorrhagic events in Covid-19 patients are not easily predictable but it may be more frequent to find them in elderly patients and with a major severity in patients with low VAT. This study has some limitations including poor patient population, poor comparison with literature data due to the relative recent spread of the disease, lack of subdivision of bleeding by site and contributing cause; for a better understanding of soft tissue bleeding in Covid patients, prospective studies and case-control analyzes with more detailed grouping are needed. Perhaps it might be interesting to know how long after the start of anticoagulant therapy and from the diagnosis of the pathology the bleeding phenomenon began.

\section{Conclusions}

our results show that soft-tissue bleeding in COVID-19 patients is more frequent and more severe in patients with low amount of visceral adipose tissue, demonstrating that the fat mass may have a containing function on bleeding by limiting its progression in surrounding structures. Other factors in our series influence the risk of bleeding, such as the age. We should also keep in mind the concomitance of anticoagulant therapy in all COVID-19 patients and the complexity of hemodynamic system in these patients, which can affect the generalization of the results.

\section{Abbreviations}

CT: Computed Tomography; TAE: Transarterial Embolization; VAT: visceral adipose tissue; SAT: subcutaneous adipose tissue.

\section{Declarations}

- Ethical Approval and Consent to participate: all procedures performed in studies involving human participants were in accordance with the ethical standards of the institutional and national research committee and with the 1964 Helsinki declaration and its later amendments or comparable ethical standards.

This article does not contain any studies with animals performed by any of the authors.

- Consent for publication: not applicable

- Availability of data and materials: not applicable

- Competing interests: not applicable

- Funding: not applicable

- Authors' contributions:

All authors declare that they have no conflict of interest. 
All authors submitted and take responsibility of this original manuscript.

All authors affirm that all contents of this manuscripts has never been published or submitted for publications elsewhere.

All authors approve the publication.

All authors retain the copyright to the publisher.

All authors refuse any financial, consultant, institutional and other relationships that might lead to bias or conflict of interest.

- Eliodoro Faiella: guarantor of integrity of the entire study, study concepts and design

- Gennaro Castiello: study concepts and design, manuscript preparation

- Domiziana Santucci: manuscript editing

- Carlo Altomare: statistical analysis

- Giuseppina Pacella: manuscript preparation

- Caterina Bernetti: literature research, manuscript preparation

- Ugo Ferrari: data collection

- Moises Muley: clinical studies

- Raffaele Antonelli Incalzi: supervision

- Bruno Beomonte Zobel: data analysis, supervision

- Rosario Francesco Grasso: supervision

- Acknowledgements: not applicable

\section{References}

1. A. S. Fauci, H. C. Lane, and R. R. Redfield, 'Covid-19 - Navigating the Uncharted', N. Engl. J. Med., vol. 382, no. 13, pp. 1268-1269, Mar. 2020, doi: 10.1056/NEJMe2002387.

2. H. Yue et al., 'Clinical characteristics of coronavirus disease 2019 in Gansu province, China', Ann. Palliat. Med., vol. 9, no. 4, pp. 1404-1412, Jul. 2020, doi: 10.21037/apm-20-887.

3. F. Zhou et al., 'Clinical course and risk factors for mortality of adult inpatients with COVID-19 in Wuhan, China: a retrospective cohort study', Lancet Lond. Engl., vol. 395, no. 10229, pp. 1054-1062, Mar. 2020, doi: 10.1016/S0140-6736(20)30566-3.

4. A. Dorgalaleh, 'Bleeding and Bleeding Risk in COVID-19', Semin. Thromb. Hemost., vol. 46, no. 7, pp. 815-818, Oct. 2020, doi: 10.1055/s-0040-1713434.

5. N. Tang, D. Li, X. Wang, and Z. Sun, 'Abnormal coagulation parameters are associated with poor prognosis in patients with novel coronavirus pneumonia', J. Thromb. Haemost. JTH, vol. 18, no. 4, pp. 844-847, Apr. 2020, doi: 10.1111/jth.14768. 
6. N. C. Chan and J. I. Weitz, 'COVID-19 coagulopathy, thrombosis, and bleeding', Blood, vol. 136, no. 4, pp. 381-383, Jul. 2020, doi: 10.1182/blood.2020007335.

7. J. A. Hippensteel, W. B. LaRiviere, J. F. Colbert, C. J. Langouët-Astrié, and E. P. Schmidt, 'Heparin as a therapy for COVID-19: current evidence and future possibilities', Am. J. Physiol.-Lung Cell. Mol. Physiol., vol. 319, no. 2, pp. L211-L217, Aug. 2020, doi: 10.1152/ajplung.00199.2020.

8. H. Al-Samkari et al., 'COVID-19 and coagulation: bleeding and thrombotic manifestations of SARSCoV-2 infection', Blood, vol. 136, no. 4, pp. 489-500, Jul. 2020, doi: 10.1182/blood.2020006520.

9. N. Musoke et al., 'Anticoagulation and bleeding risk in patients with COVID-19', Thromb. Res., vol. 196, pp. 227-230, Dec. 2020, doi: 10.1016/j.thromres.2020.08.035.

10. P. Lucatelli et al., 'Heparin-Related Major Bleeding in Covid-19-Positive Patient: Perspective from the Outbreak', Cardiovasc. Intervent. Radiol., vol. 43, no. 8, pp. 1216-1217, Aug. 2020, doi: 10.1007/s00270-020-02532-3.

11. L. Touma et al., 'Transcatheter Arterial Embolization of Spontaneous Soft Tissue Hematomas: A Systematic Review', Cardiovasc. Intervent. Radiol., vol. 42, no. 3, pp. 335-343, Mar. 2019, doi: 10.1007/s00270-018-2086-x.

12. A. A. D. Albashir, 'The potential impacts of obesity on COVID-19', Clin. Med. Lond. Engl., vol. 20, no. 4, pp. e109-e113, Jul. 2020, doi: 10.7861/clinmed.2020-0239.

13. Y. Huang et al., 'Obesity in patients with COVID-19: a systematic review and meta-analysis', Metabolism., vol. 113, p. 154378, Dec. 2020, doi: 10.1016/j.metabol.2020.154378.

14. D. Petrakis et al., 'Obesity - a risk factor for increased COVID-19 prevalence, severity and lethality (Review)', Mol. Med. Rep., vol. 22, no. 1, pp. 9-19, Jul. 2020, doi: 10.3892/mmr.2020.11127.

15. F. Sanchis-Gomar, C. J. Lavie, M. R. Mehra, B. M. Henry, and G. Lippi, 'Obesity and Outcomes in COVID-19: When an Epidemic and Pandemic Collide', Mayo Clin. Proc., vol. 95, no. 7, pp. 1445-1453, Jul. 2020, doi: 10.1016/j.mayocp.2020.05.006.

16. J. Yang, J. Hu, and C. Zhu, 'Obesity aggravates COVID-19: A systematic review and meta-analysis', J. Med. Virol., vol. 93, no. 1, pp. 257-261, Jan. 2021, doi: 10.1002/jmv.26237.

17. Y. Zhou, J. Chi, W. Lv, and Y. Wang, 'Obesity and diabetes as high-risk factors for severe coronavirus disease 2019 (Covid-19)', Diabetes Metab. Res. Rev., vol. 37, no. 2, p. e3377, Feb. 2021, doi: 10.1002/dmrr.3377.

18. A. Simonnet et al., 'High Prevalence of Obesity in Severe Acute Respiratory Syndrome Coronavirus-2 (SARS-CoV-2) Requiring Invasive Mechanical Ventilation', Obes. Silver Spring Md, vol. 28, no. 7, pp. 1195-1199, Jul. 2020, doi: 10.1002/oby.22831.

19. G. Pasquarelli-do-Nascimento, H. A. Braz-de-Melo, S. S. Faria, I. de O. Santos, G. P. Kobinger, and K. G. Magalhães, 'Hypercoagulopathy and Adipose Tissue Exacerbated Inflammation May Explain Higher Mortality in COVID-19 Patients With Obesity', Front. Endocrinol., vol. 11, p. 530, 2020, doi: 10.3389/fendo.2020.00530.

20. G. Favre et al., 'Visceral fat is associated to the severity of COVID-19', Metabolism., vol. 115, p. 154440, Feb. 2021, doi: 10.1016/j.metabol.2020.154440. 
21. F. Pan et al., 'Time Course of Lung Changes at Chest CT during Recovery from Coronavirus Disease 2019 (COVID-19)', Radiology, vol. 295, no. 3, pp. 715-721, Jun. 2020, doi:

10.1148/radiol.2020200370.

22. M. Francone et al., 'Chest CT score in COVID-19 patients: correlation with disease severity and shortterm prognosis', Eur. Radiol., pp. 1-10, Jul. 2020, doi: 10.1007/s00330-020-07033-y.

23. Y. Noumura, T. Kamishima, K. Sutherland, and H. Nishimura, 'Visceral adipose tissue area measurement at a single level: can it represent visceral adipose tissue volume?', Br. J. Radiol., vol. 90, no. 1077 , p. 20170253 , Aug. 2017, doi: 10.1259/bjr.20170253.

24. F. Pediconi et al., 'Visceral adipose tissue area predicts intensive care unit admission in COVID-19 patients', Obes. Res. Clin. Pract., vol. 15, no. 1, pp. 89-92, Feb. 2021, doi:

10.1016/j.orcp.2020.12.002.

25. C. Bonanad et al., 'The Effect of Age on Mortality in Patients With COVID-19: A Meta-Analysis With 611,583 Subjects', J. Am. Med. Dir. Assoc., vol. 21, no. 7, pp. 915-918, Jul. 2020, doi: 10.1016/j.jamda.2020.05.045.

26. C. B. Conti, S. Henchi, G. P. Coppeta, S. Testa, and R. Grassia, 'Bleeding in COVID-19 severe pneumonia: The other side of abnormal coagulation pattern?', Eur. J. Intern. Med., vol. 77, pp. 147149, Jul. 2020, doi: 10.1016/j.ejim.2020.05.002.

27. I. Bargellini et al., 'Spontaneous Bleedings in COVID-19 Patients: An Emerging Complication', Cardiovasc. Intervent. Radiol., vol. 43, no. 7, pp. 1095-1096, Jul. 2020, doi: 10.1007/s00270-02002507-4.

28. J. S. Berger and J. M. Connors, 'Anticoagulation in COVID-19: reaction to the ACTION trial', The Lancet, vol. 397, no. 10291, pp. 2226-2228, Jun. 2021, doi: 10.1016/S0140-6736(21)01291-5.

29. INSPIRATION Investigators, 'Effect of Intermediate-Dose vs Standard-Dose Prophylactic Anticoagulation on Thrombotic Events, Extracorporeal Membrane Oxygenation Treatment, or Mortality Among Patients With COVID-19 Admitted to the Intensive Care Unit: The INSPIRATION Randomized Clinical Trial', JAMA, vol. 325, no. 16, pp. 1620-1630, Apr. 2021, doi:

10.1001/jama.2021.4152.

\section{Figures}




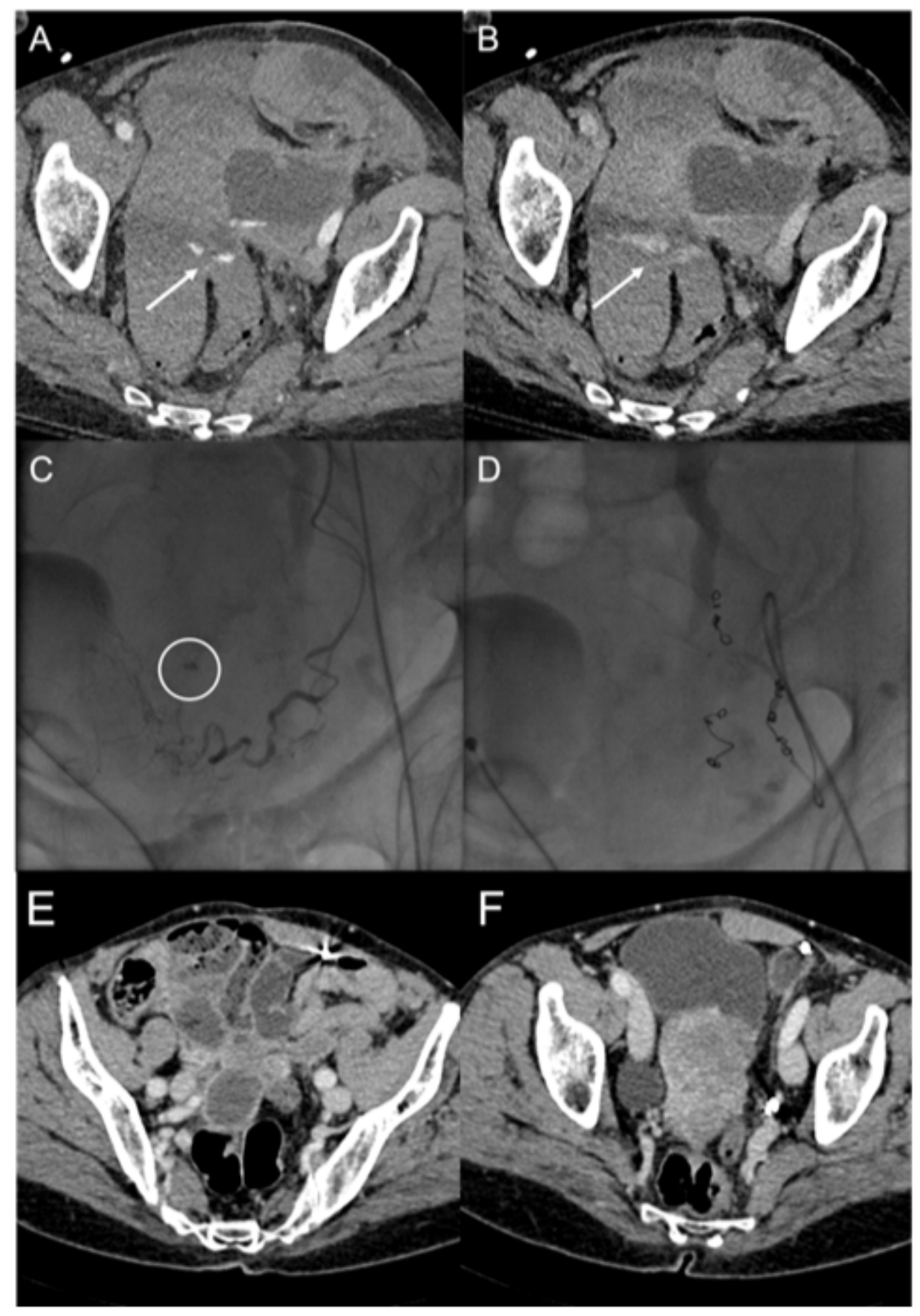

Figure 1

Bleeding in the context of the left uterine broad ligament. The CT images in A and B show the spots of active bleeding with progressive spread of contrast medium. The angiographic image in $\mathrm{C}$ shows the active bleeding spot adjacent to the left uterine artery. The angiographic image in D shows the complete embolization, by means of metallic micro-spirals, of the arterial afferents at the bleeding point. CT images in $\mathrm{E}$ and $\mathrm{F}$ show complete reabsorption of hematomas after 3 months. 


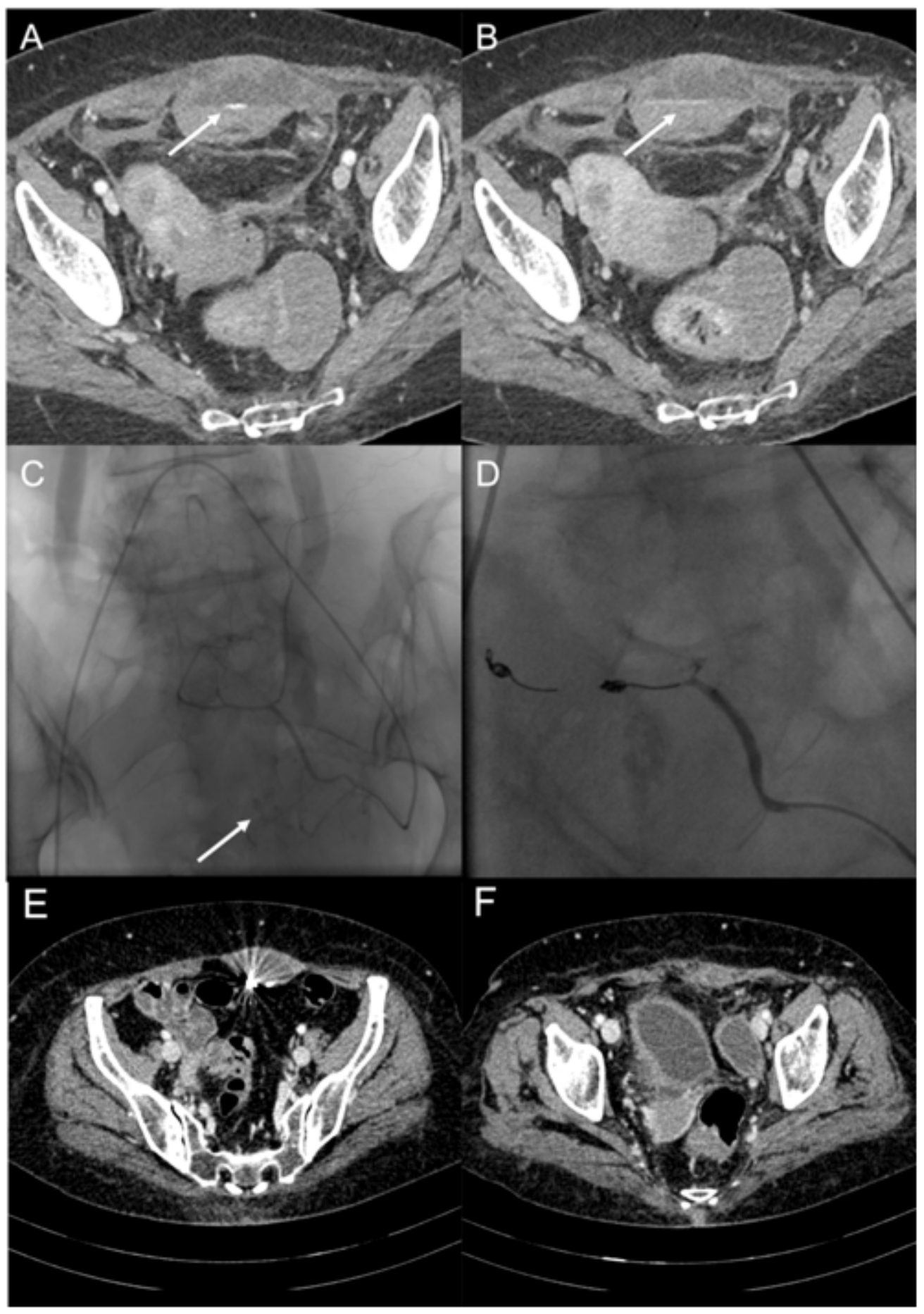

\section{Figure 2}

Bleeding in the context of the left rectus abdominis muscle. The CT images in A and B show the spots of active bleeding with progressive spread of contrast medium. The angiographic image in $\mathrm{C}$ shows the active bleeding spot adjacent to the left inferior epigastric artery. The angiographic image in $D$ shows the complete embolization, by means of glue and metallic micro-spirals, of the arterial afferents at the bleeding point. CT images in $\mathrm{E}$ and $\mathrm{F}$ show complete reabsorption of hematomas after 3 months. 

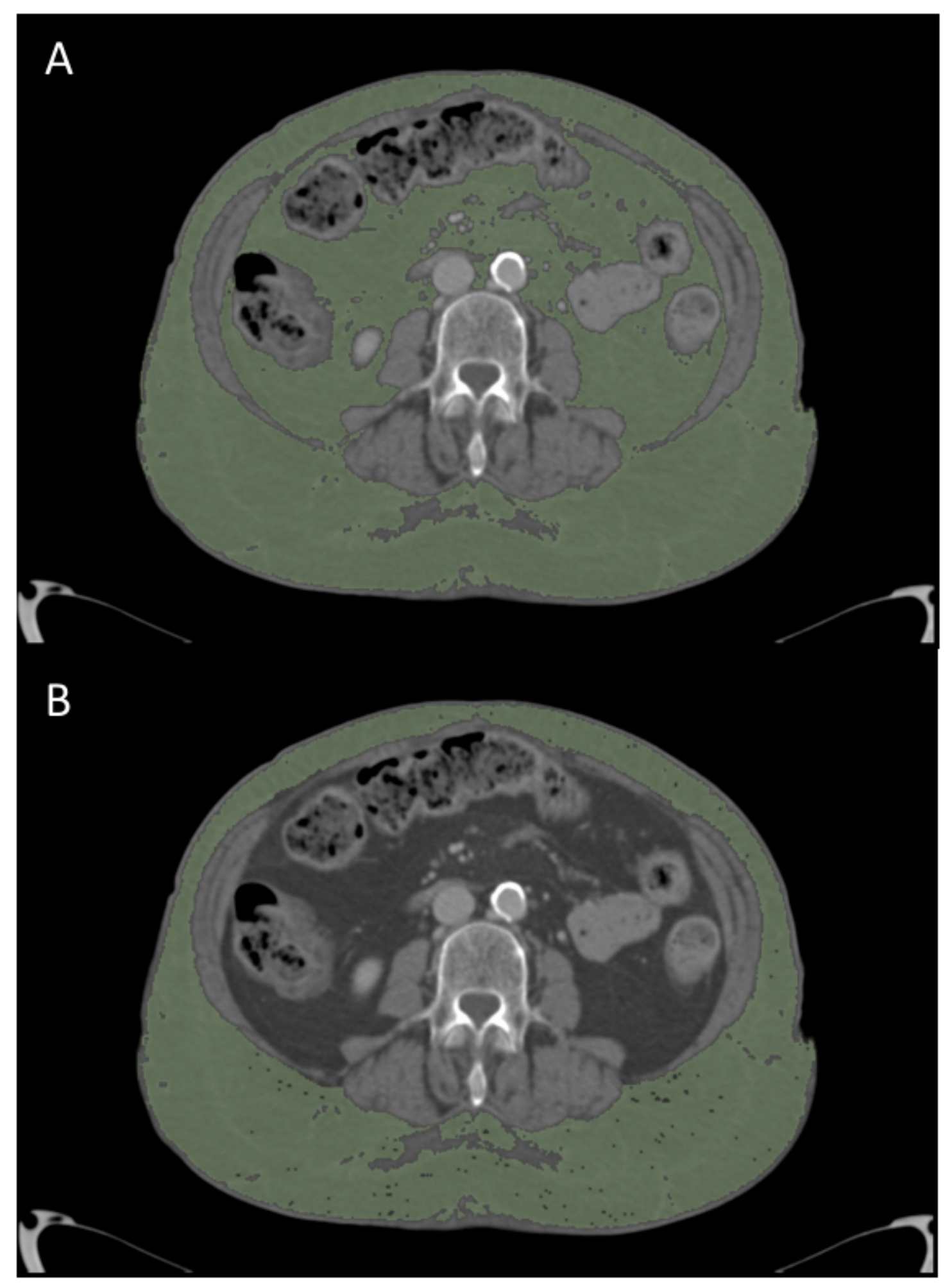

\section{Figure 3}

Measurement of adipose tissue areas in $\mathrm{cm}^{2}$ obtained analyzing axial CT images at the level of the third lumbar vertebra with an image processing application (OsiriX, Pixmeo, Bernex, Switzerland). Total adipose tissue (TAT; image in A) and subcutaneous adipose tissue (SAT; image in B) measurements; visceral adipose tissue (VAT) is calculated by difference between TAT and SAT. 